\title{
Metagenomics-Based Proficiency Test of Smoked Salmon Spiked with a Mock Community
}

\author{
Claudia Sala ${ }^{1}\left(\mathbb{D}\right.$, Hanne Mordhorst ${ }^{2}$, Josephine Grützke ${ }^{3}\left(\mathbb{D}\right.$, Annika Brinkmann ${ }^{4}$, \\ Thomas N. Petersen ${ }^{2}$, , Casper Poulsen ${ }^{2}$, Paul D. Cotter ${ }^{5}$, Fiona Crispie ${ }^{5}$, Richard J. Ellis ${ }^{6}$ \\ Gastone Castellani ${ }^{7}$, Clara Amid ${ }^{8}{ }^{\circledR}$, Mikhayil Hakhverdyan ${ }^{9}{ }^{\circledR}$, Soizick Le Guyader ${ }^{10}$, \\ Gerardo Manfreda ${ }^{11}$, Joël Mossong ${ }^{12}$, Andreas Nitsche ${ }^{4}$, Catherine Ragimbeau ${ }^{12}$, \\ Julien Schaeffer ${ }^{10}$, Joergen Schlundt ${ }^{13}$, Moon Y. F. Tay ${ }^{13}$, Frank M. Aarestrup ${ }^{2}$, \\ Rene S. Hendriksen ${ }^{2}$, Sünje Johanna Pamp ${ }^{2}$ (I) and Alessandra De Cesare ${ }^{14, *}$
}

1 Department of Physics and Astronomy, University of Bologna, 40127 Bologna, Italy; claudia.sala3@unibo.it

2 Research Group for Genomic Epidemiology, National Food Institute, Technical University of Denmark, Kemitorvet, DK-2800 Kgs, 2800 Lyngby, Denmark; hamr@food.dtu.dk (H.M.); tnpe@food.dtu.dk (T.N.P.); casper.sahl.poulsen@sund.ku.dk (C.P.); fmaa@food.dtu.dk (F.M.A.); rshe@food.dtu.dk (R.S.H.); sjpa@food.dtu.dk (S.J.P.)

3 Department of Biological Safety, German Federal Institute for Risk Assessment, 12277 Berlin, Germany; Josephine.gruetzke@bfr.bund.de

4 Highly Pathogenic Viruses, ZBS 1, Centre for Biological Threats and Special Pathogens, Robert Koch Institute, 13353 Berlin, Germany; BrinkmannA@rki.de (A.B.); NitscheA@rki.de (A.N.)

5 APC Microbiome Ireland and Vistamilk, Teagasc Food Research Centre, Moorepark, T12 YN60 Co. Cork, Ireland; paul.cotter@teagasc.ie (P.D.C.); fiona.crispie@teagasc.ie (F.C.)

6 Surveillance and Laboratory Services Department, Animal and Plant Health Agency, APHA Weybridge, Addlestone, Surrey, KT15 3NB, UK; Richard.Ellis@apha.gov.uk

7 Department of Experimental, Diagnostic and Specialty Medicine, University of Bologna, 40127 Bologna, Italy; gastone.castellani@unibo.it

8 European Molecular Biology Laboratory, European Bioinformatics Institute, Wellcome Genome Campus, Hinxton, Cambridge CB10 1SD, UK; amid@ebi.ac.uk

9 National Veterinary Institute, Ulls väg 2B, 75189 Uppsala, Sweden; mikhayil.hakhverdyan@sva.se

10 Laboratoire de Microbiologie, CEDEX 03, 44311 Nantes, France; soizick.le.guyader@ifremer.fr (S.L.G.); julien.schaeffer@ifremer.fr (J.S.)

11 Department of Agricultural and Food Sciences, University of Bologna, 40064 Ozzano dell'Emilia, Italy; gerardo.manfreda@unibo.it

12 Epidemiology and Microbial Genomics, Laboratoire National de Santé, L-3555 Dudelange, Luxembourg; joel.mossong@lns.etat.lu (J.M.); catherine.ragimbeau@lns.etat.lu (C.R.)

13 Nanyang Technological University Food Technology Centre (NAFTEC), Nanyang Technological University (NTU), 62 Nanyang Dr, Singapore 637459, Singapore; jschlundt@ntu.edu.sg (J.S.); moon.tay@ntu.edu.sg (M.Y.F.T.)

14 Department of Veterinary Medical Sciences, University of Bologna, Via Tolara di Sopra 50, 40064 Ozzano dell'Emilia, Italy

* Correspondence: alessandra.decesare@unibo.it

Received: 28 October 2020; Accepted: 23 November 2020; Published: 25 November 2020

check for updates

\begin{abstract}
An inter-laboratory proficiency test was organized to assess the ability of participants to perform shotgun metagenomic sequencing of cold smoked salmon, experimentally spiked with a mock community composed of six bacteria, one parasite, one yeast, one DNA, and two RNA viruses. Each participant applied its in-house wet-lab workflow(s) to obtain the metagenomic dataset(s), which were then collected and analyzed using MG-RAST. A total of 27 datasets were analyzed. Sample pre-processing, DNA extraction protocol, library preparation kit, and sequencing platform, influenced the abundance of specific microorganisms of the mock community. Our results highlight that despite differences in wet-lab protocols, the reads corresponding to the mock community members spiked in
\end{abstract}


the cold smoked salmon, were both detected and quantified in terms of relative abundance, in the metagenomic datasets, proving the suitability of shotgun metagenomic sequencing as a genomic tool to detect microorganisms belonging to different domains in the same food matrix. The implementation of standardized wet-lab protocols would highly facilitate the comparability of shotgun metagenomic sequencing dataset across laboratories and sectors. Moreover, there is a need for clearly defining a sequencing reads threshold, to consider pathogens as detected or undetected in a food sample.

Keywords: shotgun metagenomics; smoked salmon; proficiency test; experimentally spiked samples; wet-lab protocols

\section{Introduction}

Foodborne illnesses associated with pathogenic microorganisms are a global public health and economic challenge. In 2018, 26 European Member States reported 5146 food-borne and waterborne outbreaks with 48,365 human cases and $21.2 \%$ increase in the overall number of outbreak-related deaths, in comparison to 2017 [1]. The outbreaks with known causative agents were caused by bacteria (57.0\%), followed by bacterial toxins $(24.2 \%)$, viruses $(13.5 \%)$, other causative agents $(4.3 \%)$, and parasites $(1.0 \%)$ [1]. The techniques used for the detection and characterization of foodborne pathogens in food products evolved tremendously over the past several decades, but they generally focused on the detection of a single pathogen or just a few pathogens at a time [2]. This analytical restriction hampers the mapping of shifting microbial communities, which potentially affect the persistence of foodborne pathogens in the food production chain [2,3], and can also result in pathogens being overlooked by virtue of being novel or not being traditionally associated with a particular environment.

Shotgun metagenomics provides the potential for detection, identification, and characterization of pathogens in food [4,5], in the food chain environment [2], as well as in animals and humans [6,7]. In addition to taxonomic assignment, shotgun metagenomics also provides functional insights, through the detection of genetic markers, such as genes associated with antimicrobial resistance and virulence-related properties [5]. However, at present, deconvoluting the metagenomic data to definitively associate those markers with the same genome is still challenging $[5,8]$.

Shotgun metagenomic sequencing includes a wet-lab part, followed by sequence data analysis. Concerning the wet-lab protocols, comparative studies were performed to assess the implementation of different strategies for sample handling [7,9], nucleic acid extraction [10], library preparation [11], and sequencing [12]. Furthermore, specific assessments were done on working with contaminants [13], host DNA [14], and low biomass specimens [6], defining ideal read depths for particular biological specimens or food products, as well as defining the sequence number thresholds to confidently assign a pathogenic etiology [15]. Nowadays, Giga bases of high-quality sequence data can be easily generated at a comparatively low cost. Thus, performing high-throughput shotgun sequencing can result in a large and complex dataset from which taxonomic composition and functional capacity of the entire ecosystem under study can be determined [16].

Various pipelines for the pre-processing, assembly [17], clustering, and analyses are available for metagenomic bioinformatics, such as IMP [18], MetAMOS [19], MG-Mapper [20], MG-RAST [21], MOCAT2 [22], OneCodex [23], and RIEMS [24]. However, in some cases, the use of those pipelines requires access to high performance computing facilities, as well as laboratory personnel that is cross-trained in bioinformatics and biology, to generate the data and interpret the results obtained [8].

To access the suitability of shotgun metagenomics to detect and possibly quantify microorganisms belonging to different domain in a food sample, a proficiency test (PT) was carried out as part of the COMPARE project (www.compare-europe.eu), involving 11 participants from the EU and Singapore. All participants received the same food sample spiked with a mock community composed of six bacteria, three viruses, one parasite, and one yeast. Each participant processed the sample using in-house wet-lab 
protocol(s), up to the production of the metagenomic dataset(s). Metagenomic datasets were both submitted to a COMPARE data-hub [25] and picked up or sent directly to the participant performing the data analysis, using the MG-RAST metagenomics analysis server. The relative abundance of the microorganisms of the mock community expected in each dataset was calculated, based on the number of cells/viral genomes spiked in the food sample. Moreover, the detection of each microorganism was evaluated, considering the presence of at least 1,5 , or 10 corresponding reads enumerated in MG-RAST. The DNA metagenomic datasets were ranked according to the distance between the relative abundance of the microorganisms of the mock community in each dataset, and the expected values. The best-performing wet-lab protocols generating the metagenomic datasets closer to the expected values were then discussed.

\section{Materials and Methods}

\subsection{Samples}

An aliquot of cold smoked salmon was cut in small pieces (approximately 1-2 $\mathrm{mm}$ in width/length/depth), using a sterile scalpel and sterile petri dish. An amount of $0.2 \mathrm{~g}$ was transferred to individual sterile Nunc screw cap tubes. Subsequently, each tube was kept on ice and spiked with $50 \mu \mathrm{L}$ of a mock microbial community, consisting of six bacteria, one parasite, one fungus, and $10 \mu \mathrm{L}$ of heat-inactivated viruses (one DNA and two RNA viruses) (Table 1).

Table 1. Composition of the mock community used to spike the samples of cold smoked salmon and concentration of each microorganism. Each concentration corresponded to an expected relative abundance calculated by the number of cells of each spiked microorganism multiplied for the genome size, as indicated in the GeneBank (https:/www.ncbi.nlm.nih.gov/assembly), taking the value available or the mean ${ }^{*}$ of the different values available for the same strain. The relative abundances were obtained by normalizing the resulting values to a sum of one.

\begin{tabular}{|c|c|c|c|}
\hline Taxon (Genome Size) & $\begin{array}{l}\text { Number per Subsample } \\
\text { (Cells/Virus Genome Copies) }\end{array}$ & $\begin{array}{l}\text { Expected Relative } \\
\text { Abundance }\end{array}$ & Feature \\
\hline \multicolumn{4}{|l|}{ Bacteria } \\
\hline $\begin{array}{l}\text { Bacteroides fragilis NCTC 9343/DSM } 2151 \\
\qquad(5,241,700 \mathrm{bp})\end{array}$ & $5 \times 10^{7}$ & 0.065 & Gram - \\
\hline Escherichia coli ATCC $25922(5,166,282 *$ bp) & $5 \times 10^{7}$ & 0.064 & Gram - \\
\hline $\begin{array}{l}\text { Fusobacterium nucleatum subsp. nucleatum } \\
\text { ATCC } 25586 / \text { DSM } 15,643(2,177,300 * \mathrm{bp})\end{array}$ & $5 \times 10^{7}$ & 0.027 & Gram - \\
\hline $\begin{array}{l}\text { Propionibacterium freudenreichii subsp. } \\
\text { Freudenreichii DSM } 20271 \text { (2,649,166 bp) } \\
\text { Salmonella enterica subsp. enterica serovar }\end{array}$ & $5 \times 10^{8}$ & 0.331 & Gram + \\
\hline $\begin{array}{l}\text { Typhimurium str. ATCC 14028S/DSM } 19587 \\
\qquad(4,964,097 \text { bp })\end{array}$ & $5 \times 10^{7}$ & 0.062 & Gram - \\
\hline $\begin{array}{l}\text { Staphylococcus aureus subsp. aureus NCTC } 8325 \\
\qquad(2,821,361 \mathrm{bp})\end{array}$ & $5 \times 10^{8}$ & 0.352 & Gram + \\
\hline \multicolumn{4}{|l|}{ Viruses } \\
\hline Bovine alphaherpesvirus $1\left(135,098^{*}\right)$ & $2.41 \times 10^{9}$ & $<0.001$ & ds DNA \\
\hline Border disease virus isolate Gifhorn $(12,325 \mathrm{bp})$ & $6 \times 10^{6}$ & $<0.001$ & ssRNA \\
\hline $\begin{array}{l}\text { Bovine viral diarrhea virus type } 1 \text { isolate } \\
\text { NADL }(12,578 \mathrm{bp})\end{array}$ & $3 \times 10^{5}$ & $<0.001$ & ssRNA \\
\hline \multicolumn{4}{|l|}{ Eukaryota } \\
\hline $\begin{array}{l}\text { Cryptosporidium paroum IOWA II isolate } \\
\qquad(9,102,324 \mathrm{bp})\end{array}$ & $1 \times 10^{6}$ & 0.002 & \\
\hline Saccharomyces cerevisiae S288C $(12,157,105 \mathrm{bp})$ & $5 \times 10^{6}$ & 0.015 & \\
\hline
\end{tabular}

The tubes were kept on ice during preparation. Cell counts of the bacteria and the fungus were determined using a Petroff counting chamber, under a light microscope, counting cells in two diagonal corners on two separately prepared slides. The parasite oocysts were obtained from Waterborne Inc. in PBS, containing $1.2 \times 108$ cells (as determined by Fluorescence Activated Cell Sorting, by the 
provider). After spiking, each tube was vortex-mixed and placed at $-80{ }^{\circ} \mathrm{C}$, before shipping to each PT participant with a freezer pack kept at $-80^{\circ} \mathrm{C}$, until packaging. The frozen samples were supplemented by another $50 \mu \mathrm{L}$ of the virus mix, without additional mixing.

\subsection{Laboratory Workflows of Participants}

The wet-lab protocols used by the participants differed from each other. These are summarized in Table 2 and are detailed in the supplementary material.

A total of 27 metagenomics datasets obtained by shotgun metagenomic sequencing of spiked salmon were submitted as part of the PT. Among the 27 samples tested, 7 were not pre-processed and directly submitted to nucleic acid extraction, while 9 were pre-processed by using a bead beating protocol with TissueLyser, with modifications by each participant. Moreover, for M33 and M34, two milliliter of glycine buffer $0.05 \mathrm{M}(\mathrm{pH}$ 9) were added to the spiked salmon and homogenized in a potter grinding tube. Then, the $\mathrm{pH}$ was decreased to 3 by adding HCL. An equal volume of chloroform-butanol (v/v) was added and mixed by vortexing. After centrifugation, the supernatant was treated with Cat-Floc T (Calgon, Ellwood City, PA, USA) and precipitated with polyethylene glycol 6000 (PEG 6000) (Sigma, St. Quentin, France), for $1 \mathrm{~h}$ at $4{ }^{\circ} \mathrm{C}$, and centrifuged for $20 \mathrm{~min}$ at 11,000 $\mathrm{g}$ at $4{ }^{\circ} \mathrm{C}$. The pellet was resuspended in $2 \mathrm{~mL}$ of glycine buffer and filtrated using a cascade of $5,1.2$, and $0.45 \mathrm{~mm}$ filter pores (Minisart NML 17594, NML17593, PES16533, and PES16532). The recovered filtrates were incubated for $1 \mathrm{~h}$ at $37^{\circ} \mathrm{C}$, with 2000 Units of OmniCleave EndonucleaseTM (Lucigen corporation) and $100 \mathrm{~mL}$ of $\mathrm{MgCl} 2(100 \mathrm{mM})$. For M30, M31, and M32, the samples were centrifuged at $5000 \mathrm{rpm}$ for $20 \mathrm{~min}$ at $4{ }^{\circ} \mathrm{C}$, and the pellet was used for nucleic acid extraction. For M08, the sample was homogenized in liquid nitrogen using a ceramic mortar and pestle, followed by the gradual addition of $2.5 \mathrm{~mL}$ TE buffer. The homogenate was then centrifuged at $4000 \times \mathrm{g}$ for $10 \mathrm{~min}$ and the supernatant was filtered on a $0.45-\mu \mathrm{m}$ disc HPF Millex syringe filter (Millipore, Cork, Ireland), followed by the nuclease treatment. Subsequently, $200 \mu \mathrm{L}$ of the sample were used for DNA extraction. For M11 and M13, the samples were homogenized in liquid nitrogen, using a ceramic mortar and pestle, followed by the gradual addition of $2.5 \mathrm{~mL}$ TE buffer. The homogenate was then centrifuged at $4000 \times \mathrm{g}$ for $10 \mathrm{~min}$, and the supernatant was subjected to three cycles of the freeze-thaw method (M11: dry ice $/ 100{ }^{\circ} \mathrm{C}$; M13: dry ice $/ 37^{\circ} \mathrm{C}$ ) and submitted to DNA extraction. For M12, the sample was homogenized in liquid nitrogen, using a ceramic mortar and pestle, followed by the gradual addition of $2.5 \mathrm{~mL}$ TE buffer. The homogenate was then centrifuged at $4000 \times g$ for $10 \mathrm{~min}$, and the supernatant was filtered on a $0.45-\mu \mathrm{m}$ disc HPF Millex syringe filter (Millipore, Cork, Ireland), followed by nuclease treatment. Subsequently, $200 \mu \mathrm{L}$ of the sample was transferred to a new tube and mixed with $600 \mu \mathrm{L}$ of TRIzol Reagent (Invitrogen, Carlsbad, CA, USA), $160 \mu \mathrm{L}$ of chloroform, followed by RNA extraction. Finally, for M27 and M28, the samples were submitted to a disintegration step, using the Covaris cryoPREP CP02 [7].

For 18 samples, the target nucleic acid was DNA (Table 2). In 2 samples, the DNA was extracted using the QIAamp Fast DNA Stool Mini Kit (Qiagen, Hilden Germany) and in 3, using the DNeasy PowerSoil Microbial Kit (Qiagen). Moreover, in 5 samples, the DNA was extracted using the DNeasy PowerFood Microbial Kit (Qiagen) and in 8 samples using the QIAamp DNA Mini Kit (Qiagen) or the QIAamp UCP Pathogen Mini Kit (Qiagen). In M08, M11, and M13, the QIAamp DNA Mini Kit Qiagen was associated to Tag labeling and random amplification (SISPA) [26].

In 9 samples, the target nucleic acid was RNA (Table 2). In one sample, the RNA was extracted using the Direct-zol RNA Kit (Zimo Research) and in 2, the NucliSENS ${ }^{\circledR}$ miniMAG ${ }^{\circledR}$ (BioMérieux, Marcy l'Etoile, France). Moreover, in 2 samples, the RNA was extracted using the QIAamp Viral RNA Mini Kit (Qiagen) and in 4, the RNeasy Mini Kit (Qiagen). For M12, the use of the kit was associated to Tag labeling and Sequence-Independent, Single-Primer-Amplification (SISPA). In 5 samples, the extracted RNA was reverse transcibedusing the cDNA Synthesis System Kit (Roche, Basel, Switzerland), while in 4, the SuperScript IV Reverse Transcriptase (Invitrogen Thermo Fischer Scientific (Walthman, MA, USA) was used. 
Table 2. Metagenomic datasets and corresponding wet-lab protocols. All protocols are detailed in the Supplementary Materials. Pre-processing protocols were bead beating based protocols with TissueLyser (BBTL), no-processing (NO_PP), extraction with chloroform/butanol, Cat-Floc T, OmniCleave Endonuclease (PEGO), centrifugation (C), liquid nitrogen, centrifugation and filtration in HPF Millex filter (HCFHN), and CryoPrep Covaris (CP). The pre-processing protocols were categorized for the biostatistical analysis as no-processing (NO_PP), bead beating based protocols using TissueLyser (BBTL) and other pre-processing protocols (OTHER_PP). The DNA was extracted by QIAamp Fast DNA Stool and DNeasy Power Soil (categorized as OTHER-EXD), DNeasy Power Food Microbial Kit (PowerFood), QIAamp DNA Mini Kit with or without Sequence-Independent, Single-Primer-Amplification (SISPA), and QIAamp UCP Pathogen Mini Kit (categorized as QIAamp). The RNA was extracted by the RNeasy Mini Kit with or without TRIzol and SISPA (categorized as RNeasy Mini), QIAamp Viral RNA Mini Kit (QIAampV), NucliSENS ${ }^{\circledR}$ miniMAG ${ }^{\circledR}$ and Direct-zol RNA Kit (categorized as OTHER_EXR). The cDNA was generated using the cDNA Synthesis System Kit (cDNA SS) or the SuperScript IV Reverse Transcriptase (SS IV RT). The libraries were prepared by Nextera ${ }^{\mathrm{TM}}$ XT DNA Library Prep kit, Nextera ${ }^{\mathrm{TM}}$ DNA Flex Library Preparation kit (categorized as NexteraXT), NEBNext ${ }^{\circledR}$ Ultra ${ }^{\mathrm{TM}}$ II DNA Library Prep Kit for Illumina ${ }^{\circledR}$, TruSeq ${ }^{\circledR}$ DNA Library Prep Kit and GeneRead DNA Library kit (categorized as OTHER_L). The sequencing platforms were NextSeq500, HiSeq2500 (categorized as NextSeq500), MiniSeq, MiSeq, and Ion Torrent S5XL (categorized as OTHER_SP).

\begin{tabular}{|c|c|c|c|c|c|c|c|c|c|c|c|c|c|c|}
\hline $\begin{array}{l}\text { Metagenome } \\
\text { Dataset }\end{array}$ & $\begin{array}{c}\text { Nucleic } \\
\text { Acid }\end{array}$ & Pre-Processing & $\begin{array}{c}\text { Category } \\
\text { Label }\end{array}$ & Extraction Kit & $\begin{array}{c}\text { Category } \\
\text { Label }\end{array}$ & $\begin{array}{c}\text { cDNA } \\
\text { Generation }\end{array}$ & Library Kit & $\begin{array}{c}\text { Category } \\
\text { Label }\end{array}$ & $\begin{array}{l}\text { Sequencing } \\
\text { Strategy }\end{array}$ & $\begin{array}{c}\text { Read } \\
\text { Length }\end{array}$ & $\begin{array}{c}\text { Sequencing } \\
\text { Platform }\end{array}$ & $\begin{array}{c}\text { Category } \\
\text { Label }\end{array}$ & Gbp & $\begin{array}{c}\text { Workflow } \\
\text { Label }\end{array}$ \\
\hline M06 & DNA & BBTL & BBTL & QIAamp Fast DNA Stool & OTHER_EXD & & NexteraXT & NexteraXT & Paired-end & 120 & NextSeq 500 & NextSeq 500 & 1.34 & WF1 \\
\hline M15 & DNA & BBTL & BBTL & QIAamp Fast DNA Stool & OTHER_EXD & & NexteraXT & NexteraXT & Paired-end & 120 & NextSeq 500 & NextSeq 500 & 2.2 & WF1 \\
\hline M24 & DNA & NO_PP & NO_PP & DNeasy PowerSoil & OTHER_EXD & & NexteraXT & NexteraXT & Paired-end & 150 & NextSeq 500 & NextSeq 500 & 8.95 & WF2 \\
\hline M29 & DNA & NO_PP & NO_PP & DNeasy PowerSoil & OTHER_EXD & & Nextera Flex & NexteraXT & Paired-end & 150 & MiniSeq & OTHER_SP & 3.43 & WF2 \\
\hline M38 & DNA & NO_PP & NO_PP & DNeasy PowerSoil & OTHER_EXD & & NexteraXT & NexteraXT & Paired-end & 150 & NextSeq 500 & NextSeq 500 & 12.2 & WF2 \\
\hline M33 & RNA & PEGO & OTHER_PP & NucliSENS MiniMag & OTHER_EXR & SS IV RT & NEBNext & OTHER_L & Paired-end & 150 & MiSeq & OTHER_SP & 0.92 & WF3 \\
\hline M34 & RNA & PEGO & OTHER_PP & NucliSENS MiniMag & OTHER_EXR & SS IV RT & NEBNext & OTHER_L & Paired-end & 150 & Miseq & OTHER_SP & 0.86 & WF3 \\
\hline M16 & DNA & BBTL & BBTL & DNesasy PowerFood & PowerFood & & NexteraXT & NexteraXT & Paired-end & 120 & NextSeq 500 & NextSeq 500 & 1.83 & WF4 \\
\hline M18 & DNA & BBTL & BBTL & DNesasy PowerFood & PowerFood & & NexteraXT & NexteraXT & Paired-end & 120 & NextSeq 500 & NextSeq 500 & 2.50 & WF4 \\
\hline M30 & DNA & C & OTHER_PP & DNesasy PowerFood & PowerFood & & NexteraXT & NexteraXT & Paired-end & 150 & NextSeq 500 & NextSeq 500 & 7.97 & WF6 \\
\hline M31 & DNA & $\mathrm{C}$ & OTHER_PP & DNesasy PowerFood & PowerFood & & NexteraXT & NexteraXT & Paired-end & 150 & NextSeq 500 & NextSeq 500 & 9.81 & WF6 \\
\hline M32 & DNA & C & OTHER_PP & DNesasy PowerFood & PowerFood & & NexteraXT & NexteraXT & Paired-end & 150 & NextSeq 500 & NextSeq 500 & 8.44 & WF6 \\
\hline M07 & DNA & NO_PP & NO_PP & QIAamp UCP Pathogen & QIAamp & & NexteraXT & NexteraXT & Paired-end & 120 & NextSeq 500 & NextSeq 500 & 1.03 & WF7 \\
\hline M10 & DNA & NO_PP & NO_PP & QIAamp UCP Pathogen & QIAamp & & NexteraXT & NexteraXT & Paired-end & 120 & NextSeq 500 & NextSeq 500 & 1.77 & WF7 \\
\hline M26 & DNA & BBTL & BBTL & QIAamp & QIAamp & & TruSeq & OTHER_L & Paired-end & 150 & NextSeq 500 & NextSeq 500 & 12.2 & WF8 \\
\hline M36 & DNA & BBTL & BBTL & QIAamp & QIAamp & & TruSeq & OTHER_L & Paired-end & 250 & HiSeq 2500 & NextSeq 500 & 8.88 & WF8 \\
\hline M25 & RNA & NO_PP & NO_PP & QIAamp Viral RNA & QIAamp & cDNA SS & NexteraXT & NexteraXT & Paired-end & 150 & NextSeq 500 & NextSeq 500 & 8.64 & WF9 \\
\hline M37 & RNA & NO_PP & NO_PP & QIAamp Viral RNA & QIAamp & SS IV RT & NexteraXT & NexteraXT & Paired-end & 200 & Miseq & OTHER_SP & 8.84 & WF10 \\
\hline M08 & DNA & HCFHN & OTHER_PP & QIAamp + SISPA & QIAamp & & NexteraXT & NexteraXT & Paired-end & 300 & MiSeq & OTHER_SP & 1.99 & WF11 \\
\hline M11 & DNA & HCFHN & OTHER_PP & QIAamp + SISPA & QIAamp & & NexteraXT & NexteraXT & Paired-end & 300 & Miseq & OTHER_SP & 2.07 & WF11 \\
\hline M27 & DNA & $\mathrm{CP}$ & OTHER_PP & QIAamp & QIAamp & & GeneRead & OTHER_L & Single-end & 250 & Ion Torrent S5XL & OTHER_SP & 2.14 & WF12 \\
\hline M19 & RNA & BBTL & BBTL & RNeasy Mini kit & RNeasy Mini & cDNA SS & NexteraXT & NexteraXT & Paired-end & 120 & NextSeq 500 & NextSeq 500 & 4.95 & WF13 \\
\hline M20 & RNA & BBTL & BBTL & RNeasy Mini kit & RNeasy Mini & cDNA SS & NexteraXT & NexteraXT & Paired-end & 120 & NextSeq 500 & NextSeq 500 & 5.06 & WF13 \\
\hline M12 & RNA & HCFHN & OTHER_PP & RNeasy Mini + SISPA & RNeasy Mini & SS IV RT & NexteraXT & NexteraXT & Paired-end & 300 & Miseq & OTHER_SP & 2.63 & WF14 \\
\hline M28 & RNA & $\mathrm{CP}$ & OTHER_PP & RNeasy Mini kit & RNeasy Mini & cDNA SS & GeneRead & OTHER_L & Single-end & 250 & Ion Torrent S5XL & OTHER_SP & 2.25 & WF15 \\
\hline
\end{tabular}


For the library preparation, 19 samples were processed using the Nextera ${ }^{\mathrm{TM}}$ XT DNA Library Prep kit (Illumina) and in one, the Nextera ${ }^{\mathrm{TM}}$ DNA Flex Library Preparation kit (Illumina, San Diego, CA, USA) was used. The libraries for the remaining 7 samples were prepared using the TruSeq ${ }^{\circledR}$ DNA Library Prep Kit Illumina $(n=3)$, the NEBNext ${ }^{\circledR}$ Ultra ${ }^{\mathrm{TM}}$ II DNA Library Prep Kit for Illumina ${ }^{\circledR}$ (New England BioLabs, Ipswich, MA, USA) $(n=2)$, and the GeneRead DNA Library kit Qiagen $(\mathrm{n}=2)$ (Table 2).

All samples were sequenced in paired-end, except two (Table 2), and the read lengths were achieved by sequencing ranged between 120 and $300 \mathrm{bp}$ (Table 2). A total of 16 samples were sequenced on the NextSeq500 (Illumina), while the others were sequenced on the HiSeq2500 (Illumina) $(n=1)$, MiSeq (Illumina) ( $\mathrm{n}=7$ ), MiniSeq (Illumina) $(\mathrm{n}=1)$, and Ion S5XL System (Thermo Fischer Scientifics) $(\mathrm{n}=2)$ (Table 2). The sequencing output associated with each dataset ranged between 0.86 and $12.2 \mathrm{Gbp}$.

\subsection{Data Sharing}

All metagenomic datasets included in this PT are publicly available on https://www.mg-rast.org/ mgmain.html?mgpage=project\&project=mgp86519 in the MG-RAST server, under the project labelled as Food metagenomic ring trial 2018. The metadata associated with the metagenomic datasets are those detailed in Table 2.

\subsection{Bioinformatics and Statistical Analysis}

The workflows including DNA as the target nucleic acid were exploited in the modelling and bio-statistical analysis described below, while RNA-based metagenomic datasets were only used to evaluate the detection performances, since the lack of information on RNA copy numbers prevented the possibility of computing quantitative comparisons. For the bio-statistical analysis, similar wet-lab steps were categorized together (Table 2).

Filtering, trimming, and taxonomic classification of raw reads were performed using MG-RAST (https://www.mg-rast.org) [27] and the RefSeq reference database [28]. The statistical analysis was performed in R, v3.5.1, using the libraries phyloseq v1.26.1 [29] and DESeq2 v1.22.1 [30].

Before proceeding with the statistical analysis, the read counts were normalized using DESeq2 [30], taking into account the compositional nature of the data. In brief, starting from the whole read counts table, DESeq2 estimates the size factor of each sample, as the median of the ration of the observed counts to those of a reference sample, obtained by taking the geometric mean across samples. Then, the normalized counts are obtained by dividing the original counts by the estimated size factors. Finally, DESeq2 computes the dispersion estimates of each gene using an Empirical Bayes approach. Such estimates are then exploited in the negative binomial generalized linear models used in the differential analysis [30,31].

The expected proportions of the microorganisms in the mock community and those detected in the metagenomics datasets submitted as part of the PT, were compared using the following approach. The expected relative abundances of the mock community were calculated, based on the cell counts of each spiked microorganism (Table 1). Such concentrations were first multiplied by the genome length to simulate the fact that in a sequencing experiment, longer genomes would be represented by a higher number of reads. Then, relative abundances were obtained by normalizing the resulting values to sum of one. The empirical relative abundances obtained in the 27 DNA metagenomic datasets were computed, based on the number of reads that mapped on the mock community microorganisms. Specifically, for each sample, the normalized abundances were first obtained with DESeq2 and the relative abundances of the mock community microorganisms were computed by normalizing the sum to one such abundances.

To visually compare the expected and the experimentally obtained compositions of the mock community in the DNA metagenomic datasets, we plotted the relative abundances in the form of bar plots. Differences in the mock community composition among samples and between each sample and the expected composition, were also evaluated by computing the Bray-Curtis beta-diversity displayed 
using the heat map. Samples were then ranked based on their distance (i.e., Bray-Curtis beta-diversity) from the expected composition of the mock community.

The statistical comparison of the species abundances obtained with different experimental parameters was performed with DESeq2. Specifically, starting from the normalized read counts, DESeq2 was used to compute the negative binomial generalized linear models for each taxon and, hence, to test the impact of the variables of interest.

We evaluated a multiple regression model in which we considered all experimental parameters in order to detect the effect of each variable, after adjustment for all others. To select the model variables, we first estimated the relationship between the variables using Fisher's exact test for categorical data and the slope of a linear regression model for numerical variables. The only pair of variables for which the $p$-value was $<0.0001$ was read length-sequencing platform $\left(p\right.$-value $\left.=4.47 \times 10^{-5}\right)$. In all other cases, the $p$-value was $\geq 0.0001$ and both variables were hence included in the model. All in all, the tested variables were pre-processing protocol, DNA extraction kit, library preparation strategy, sequencing depth, and sequencing platform. The advantage of using a multiple regression model that considers all predictors (experimental variables) at the same time is that, when evaluating the effect of one predictor, it adjusts for the effect of all others. The resulting estimates of this model, sometimes called 'partial effects', hence, indicate the effect that each experimental variable has on the abundances of a certain species, when all other variables are fixed. Statistical significance was assessed using the Likelihood Ratio Test for multiple comparisons and the Wald's test for pairwise comparisons. Moreover, the impact of each workflow on the detected mock community microorganisms' abundances was evaluated by performing a pairwise two-samples $t$-test between the DESeq2 normalized read counts, obtained with each pair of workflows. For bacteria, the parasite and the yeast, such a $t$-test was computed by considering the mean value and the variance of the abundances detected in the workflows' samples. Since only one sample was tested by applying WF12, in this case, the sample mean of each species was set to the value of its abundance in that sample and the sample variance was estimated as the average of the variances observed for that species, in all other workflows. Finally, the sample size was set to the average number of samples present in the other workflows. In this approximated test, we assume that the variance of each microorganism's abundance among the samples within a workflow is the same for all workflows with only small deviations (i.e., variances of the microorganisms' abundances are comparable between different workflows), as shown in Figure S1. In all tests, the p-values were adjusted for multiple testing using the Benjamini-Hochberg procedure [32] and a significance level of 0.05 was used to identify the statistically significant differences.

\section{Results}

\subsection{Relative Abundance of the Reads Assigned to the Taxonomic Domains and the Microorganisms of the Mock Community}

The reads belonging to different taxonomic domains were quantified in the 27 metagenomics datasets, submitted as part of the PT (Table 3).

The percentage of reads assigned to Eukaryota, including the two microorganisms belonging to the mock community (i.e., C. parvum and S. cerevisiae) ranged between $2.957 \%$ (M34) and $80.567 \%$ (M11). The reads assigned to the domain bacteria ranged between $18.823 \%$ (M11) and 95.202\% (M36), and those assigned to viruses ranged between $0.031 \%$ (M19) to $38.344 \%$ (M33) (Table 3). These results showed that the reads assigned to each domain largely differed among the metagenomic datasets and this affected the reads assigned to the microorganisms of the mock community. While it is difficult to explain each of these differences, in the three datasets in which the percentage of reads assigned to the viruses was $\leq 0.1$ (i.e., M19, M20, and M28), RNA was extracted using the RNeasy mini kit. 
Table 3. Number of reads in each metagenomic dataset obtained from spiked salmon. For each dataset, the percentage of reads assigned to Eukaryota, Bacteria, Viruses, Archaea, the number of reads belonging to the microorganisms of the mock community, and their percentage in relation to the total number of reads are detailed.

\begin{tabular}{|c|c|c|c|c|c|c|}
\hline $\begin{array}{c}\text { Metagenomic Dataset } \\
\text { Label }\end{array}$ & N. Reads & \% Eukaryota & $\%$ Bacteria & $\%$ Viruses & $\%$ Archaea & $\begin{array}{l}\text { N. Reads Mock } \\
\text { Community (\%) }\end{array}$ \\
\hline M06 & $1,644,354$ & 14.631 & 85.004 & 0.319 & 0.025 & $875,934(53.27)$ \\
\hline M07 & 847,827 & 20.857 & 78.846 & 0.249 & 0.036 & $369,389(43.57)$ \\
\hline M08 & 742,029 & 80.338 & 19.050 & 0.325 & 0.286 & $20,440(2.76)$ \\
\hline M10 & $1,071,120$ & 25.539 & 74.097 & 0.326 & 0.028 & $388,946(36.31)$ \\
\hline M11 & 730,610 & 80.567 & 18.823 & 0.309 & 0.299 & $3763(0.52)$ \\
\hline M12 & 537,347 & 79.502 & 19.747 & 0.469 & 0.264 & $11,073(2.06)$ \\
\hline M13 & 993,696 & 72.565 & 26.222 & 0.488 & 0.725 & $10,600(1.07)$ \\
\hline M15 & $1,508,927$ & 21.857 & 77.846 & 0.254 & 0.033 & $635,158(42.09)$ \\
\hline M16 & $1,206,052$ & 24.369 & 75.370 & 0.216 & 0.036 & $496,223(41.14)$ \\
\hline M18 & $1,867,262$ & 19.308 & 80.394 & 0.259 & 0.027 & $681,708(35.61)$ \\
\hline M19 & 16,187 & 31.951 & 67.770 & 0.031 & 0.043 & $826(5.1)$ \\
\hline M20 & 16,702 & 64.459 & 35.475 & 0.048 & 0.018 & 1225 (7.33) \\
\hline M23 & 82,614 & 45.051 & 54.713 & 0.171 & 0.036 & $21,891(26.50)$ \\
\hline M24 & $3,304,160$ & 31.519 & 68.311 & 0.117 & 0.046 & $1,373,626(41.57)$ \\
\hline M25 & $1,173,758$ & 45.668 & 54.015 & 0.222 & 0.073 & $353,797(30.14)$ \\
\hline M26 & $4,803,071$ & 40.370 & 59.363 & 0.230 & 0.028 & $1,332,603(27.75)$ \\
\hline M27 & 911,713 & 46.621 & 53.249 & 0.104 & 0.021 & $205,421(22.53)$ \\
\hline M28 & 2340 & 72.393 & 27.564 & 0.043 & 0 & $203(8.68)$ \\
\hline M29 & $1,507,815$ & 35.777 & 64.055 & 0.115 & 0.045 & $430,397(28.54)$ \\
\hline M30 & $3,680,106$ & 14.271 & 85.576 & 0.122 & 0.022 & $2,217,739(60.26)$ \\
\hline M31 & $3,360,140$ & 17.916 & 81.949 & 0.104 & 0.021 & $1,883,366(56.05)$ \\
\hline M32 & $4,884,497$ & 11.043 & 88.809 & 0.119 & 0.020 & $2,769,020(56.69)$ \\
\hline M33 & 203,116 & 15.636 & 45.945 & 38.344 & 0.072 & $2156(1.06)$ \\
\hline M34 & 267,871 & 2.957 & 93.85 & 3.017 & 0.084 & $2231(0.83)$ \\
\hline M37 & $1,735,966$ & 48.139 & 51.513 & 0.265 & 0.076 & $511,159(29.45)$ \\
\hline M36 & $17,120,850$ & 4.586 & 95.202 & 0.148 & 0.057 & $9,405,164(54.93)$ \\
\hline M38 & $5,803,430$ & 21.492 & 78.322 & 0.135 & 0.044 & $2,329,995(40.15)$ \\
\hline
\end{tabular}

The quantification of the mock community microorganisms, in terms of relative abundance, differed among the DNA metagenomic datasets (Table S1). As detailed in Materials and Methods, the expected relative abundance of each microorganism was calculated, based on the number of cells and virus genome copies experimentally spiked in the salmon, and the microorganisms' genome size. The relative abundances obtained from the 18 metagenomic datasets were compared to each other and to the expected one (Figure 1), and the pairwise Bray-Curtis dissimilarity among samples and with the expected composition was computed (Figure 2).

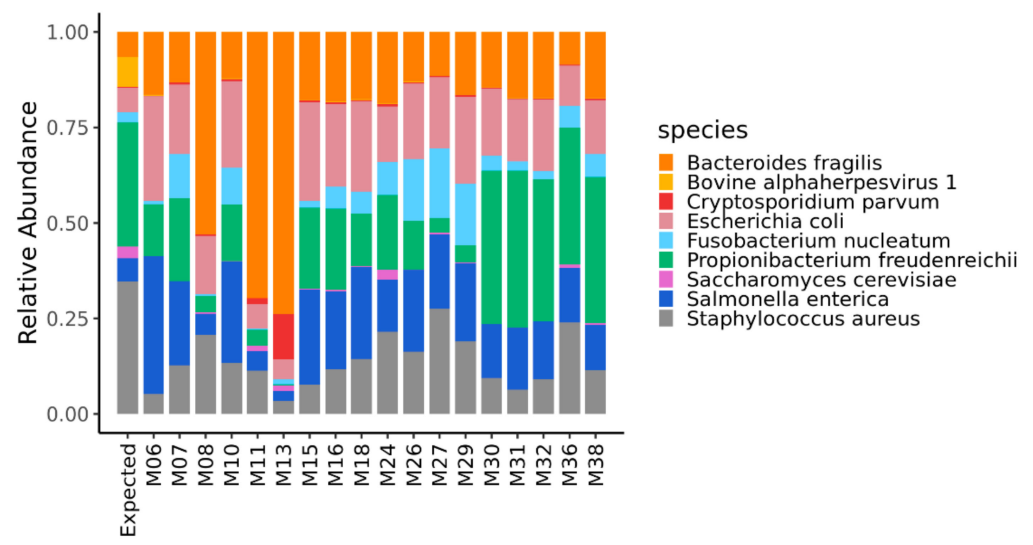

Figure 1. Relative abundance of the microorganisms of the mock community quantified in the DNA metagenomic datasets obtained from spiked salmon. The first bar in the figure refers to the expected relative abundance for the microorganisms experimentally spiked in the salmon. 


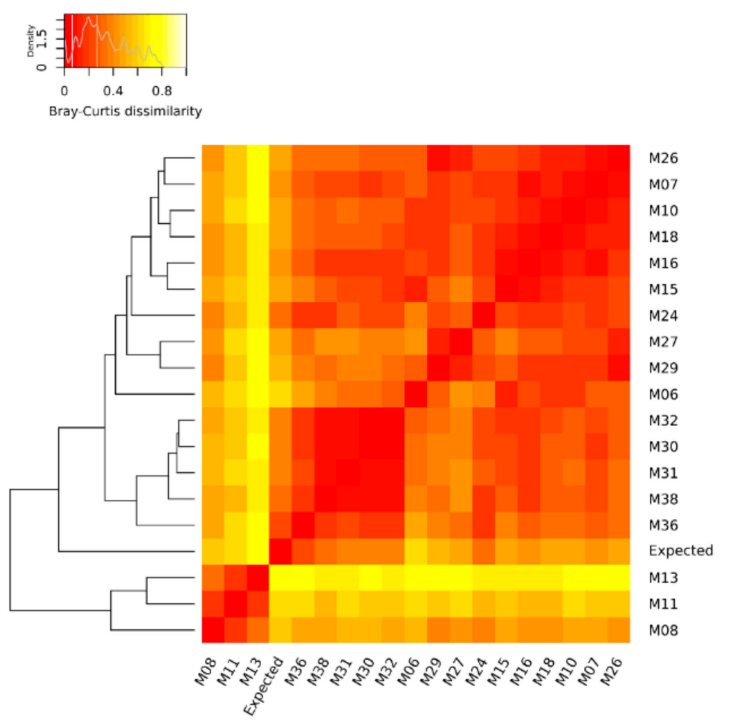

Figure 2. Heat map of Bray-Curtis dissimilarity and average linkage clustering dendrogram showing the similarity between the benchmark (expected) and the DNA metagenomic datasets obtained from spiked salmon, considering the microorganisms of the mock community. In the heat map, when a cell is colored in red, it indicates that the two associated samples (in the corresponding row and column) have an identical composition, while a white cell refers to two samples with the most different composition.

Table S2 shows the reads enumerated for each microorganism of the mock community using MG-RAST. The read number threshold to consider a microorganism as detected or undetected in a metagenomic dataset is currently unknown. Nevertheless, Table S3 summarizes the percentage of metagenomic datasets obtained from DNA and RNA in which each microorganism of the mock community was detected by assessing the enumeration in MG-RAST of at least 1,5, or 10 corresponding reads, as the detection threshold. All spiked bacteria, the yeast, and the parasite, were always detected in the metagenomic datasets from DNA, while the percentage of metagenomic datasets in which C. parvum was detected decreased from 100 to $67 \%$ in the metagenomic dataset from RNA, increasing the detection threshold from one to 10 reads. The same result applied to F. nucleatum and P. freudenreichii detected in $100 \%$ of the metagenomic datasets from RNA, by considering one read as the detection threshold, while in $78 \% 10$ reads were considered as the detection threshold (Table S3). In relation to viruses, the DNA virus was detected in $83 \%$ of the metagenomic datasets from DNA but also in 56 to $33 \%$ of those from RNA. On the contrary, the RNA viruses were hardly detected in RNA metagenomic datasets, when even 1 read was considered as the detection threshold, and it did not show up at all in the DNA metagenomic datasets (Table S3).

3.2. Ranking of the Metagenomic Datasets Based on Their Dissimilarity to the Expected Composition and Assessment of the Impact of Each Variable of the Workflow on the Abundance of the Mock Community Members

Considering the relative abundances of all microorganisms of the mock community, the DNA metagenomic datasets were ranked according to their Bray-Curtis distance from the expected values. The metagenomic dataset ranking closest to the expected value was M36 (Bray-Curtis distance 0.199) followed by M38 (Table 4), and this ranking did not change when considering the bacteria only (i.e., M36 Bray-Curtis distance 0.152) (Table S4). 
Table 4. Ranking of the DNA metagenomic datasets obtained testing spiked salmon in relation to their similarity to the expected composition of the mock community. The distance from the expected mock community composition is measured as Bray-Curtis dissimilarity.

\begin{tabular}{ccc}
\hline Metagenomic Dataset & Distance from the Mock Community & Rank \\
\hline M36 & 0.207 & 1 \\
M38 & 0.337 & 2 \\
M24 & 0.345 & 3 \\
M30 & 0.361 & 4 \\
M32 & 0.370 & 5 \\
M31 & 0.395 & 6 \\
M07 & 0.435 & 7 \\
M16 & 0.447 & 8 \\
M27 & 0.464 & 9 \\
M26 & 0.488 & 10 \\
M18 & 0.495 & 11 \\
M10 & 0.496 & 12 \\
M15 & 0.498 & 13 \\
M29 & 0.544 & 14 \\
M08 & 0.556 & 15 \\
M06 & 0.610 & 16 \\
M11 & 0.646 & 17 \\
M13 & 0.790 & 18 \\
\hline
\end{tabular}

Among the variables investigated in the workflows, those impacting on the abundance of one or more microorganisms spiked in the salmon were the pre-processing protocol, the DNA extraction protocol, the library preparation strategy, and the sequencing platform.

The pre-processing protocol significantly affected the detected abundances of C. parvum, E. coli, F. nucleatum, P. freudenreichii, S. enterica, and S. aureus (Table S5, Figure S2A). The normalized mean abundances of the parasite and F. nucleatum were significantly higher in the metagenomic datasets obtained without pre-processing, in comparison to where BBTL was applied, while the application of a bead beating protocol provided the best results in terms of E. coli, P. freudenreichii, S. enterica, and S. aureus abundances detected (Table S5, Figure S2A). Moreover, for F. nucleatum, the application of any pre-processing protocol worked better than all the other tested pre-processing protocols (Table S5, Figure S2A).

The abundances of C. paroum, B. fragilis, E. coli, P. freudenreichii, S. enterica, and S. aureus were significantly higher in the metagenomic datasets where the DNA was extracted by PowerFood, in comparison to the QIAamp DNA Mini Kit, with or without SISPA or the QIAamp UCP Pathogen Mini Kit, while for F. nucleatum, the latter performed better (Table S6, Figure S2B). On the other hand, for both F. nucleatum and S. aureus, the QIAamp DNA Mini Kit, with or without SISPA or the QIAamp UCP Pathogen Mini Kit, performed better than QIAamp Fast DNA Stool Mini Kit and the DNeasy PowerSoil Microbial Kit, while for B. fragilis, E. coli, P. freudenreichii, and S. enterica, it was the opposite (Table S6, Figure S2B).

The abundances of E. coli, F. nucleatum, P. freudenreichii, S. enterica, and S. aureus were significantly affected by the library preparation protocol. Nextera ${ }^{\mathrm{TM}}$ XT DNA Library Prep kit and Nextera ${ }^{\mathrm{TM}}$ DNA Flex Library Preparation kit worked better for E. coli, while TruSeq ${ }^{\circledR}$ DNA Library Prep Kit, NEBNext ${ }^{\circledR}$ Ultra $^{\mathrm{TM}}$ II DNA Library Prep Kit for Illumina ${ }^{\circledR}$ and the GeneRead DNA Library kit, resulted in better detected abundances for F. nucleatum, P. freudenreichii, S. enterica, and S. aureus (Table S7, Figure S2C).

The yeast abundance was not affected by the sequencing platform, while the abundances of all other microorganisms was significantly higher in the metagenomic datasets obtained by sequencing on a NextSeq500 or a HiSeq 2500, in comparison to other sequencing platforms (i.e., MiSeq, MiniSeq, Ion S5XL System) (Table S8, Figure S2D). 


\subsection{Assessment of the Impact of Each Workflow on the Abundance of the Mock Community Members}

The results for each workflow (WF), starting with DNA as a target nucleic acid were compared by performing a pairwise approximated t-test, in which we tested if the mock community microorganism's abundances detected by each pair of workflows differed.

The average abundance of the parasite was significantly higher for the samples processed using the WFs 2 and 6, in comparison to WF11 (Tables S9 and S10, Figure S3). In the WF2, the salmon is transferred to a Power Bead Tube inserted into the preheated $\left(55^{\circ} \mathrm{C}\right)$ adapter of TissueLyser II and bead-beating was applied, before centrifugation at full speed for $1 \mathrm{~min}$. Subsequently, the supernatant was transferred to a new $2 \mathrm{~mL}$ tube before DNA extraction, using the MoBio PowerFood Microbial DNA Isolation kit. In the WF6, the salmon was centrifuged at $5000 \mathrm{rpm}$ for $20 \mathrm{~min}$ at $4{ }^{\circ} \mathrm{C}$, and then processed as in WF2. The main differences between WF11 in comparison to WFs 2 and 6 was that sequencing was performed on the longer reads (i.e., 300 vs. $150 \mathrm{bp}$ ) and, on average, at a lower depth ( 2 vs. $>7 \mathrm{Gbp}$ ). Moreover, as detailed in the supplementary material, the sample handling protocol in WF11 was very long and might have negatively impacted the DNA recovery. The significantly low performances of WF11, in comparison to all other WFs, was also quantified for F. nucleatum. The average abundance of the yeast was significantly higher for the samples processed using WF4, including a bead beating protocol with TissueLyser than for WF6, while for B. fragilis it was the opposite (Tables S9 and S10, Figure S3).

\section{Discussion}

Shotgun metagenomics is a culture-independent methodology with potential to contribute to food-borne outbreaks detection and risk assessment of food-borne pathogens [33]. It consists of a wet-lab part and a dry-lab part. The wet-lab part includes the sample collection for the generation of the raw sequencing dataset. The dry-lab part includes the bioinformatic analysis, resulting in taxonomic and functional gene assignments, using reference or de novo strategies, and the biostatistics analysis, translating the bioinformatic results in biologically meaningful observations. Many publications are available on different bioinformatic strategies to trim, assemble, and assign reads and contigs, to known or unknown taxonomic and functional entities [34]. However, there are few fully accessible and transparent bioinformatic workflows and many of the online available bioinformatic tools for non-experts are not constantly updated in the reference databases. Biostatistics strategies are complex and thus they must be selected with great care, when modelling and analyzing the metagenomic datasets. Therefore, the pipelines fitting specific scenarios and case studies should be implemented and shared at an international level, to improve harmonization in metagenomic dataset analysis and interpretation.

This PT was organized with the aim to compare the suitability of different metagenomic wet-lab protocols, for both detecting and quantifying the relative abundance of microorganisms belonging to the different domains that were experimentally spiked in cold smoked salmon. Although a drawback of this study was that the large number of variables included in the wet-lab workflows applied by the participants resulted in the need to categorize similar but not identical wet-lab steps, nonetheless, this PT was the first exercise on a real food matrix, while other studies addressed artificial datasets. Moreover, to make the results of this PT as comprehensive as possible, the pipeline used for the bioinformatic analysis (i.e., MG-RAST) is a freely available and user-friendly web resource, while more powerful bioinformatic tools could have been used. The outputs of this PT should contribute to speed up the application of shotgun metagenomic in food microbiome studies and in food safety risk assessment, but for both, a quality assurance approach consistent with regulatory standards is mandatory. At present, individual research institutes set the internal reference standards, but the lack of universal reference organisms and genomic material makes it difficult to compare assay performances between different laboratories [35]. Hence, it is unlikely that shotgun metagenomic sequencing will replace the culture methods for foodborne pathogen investigation in the near future, although its great potential was demonstrated $[5,36,37]$. 
The main methodological constraints to be overcome are the lack of harmonized and validated methods, the low sensitivity in detecting certain taxa in the sample, or the fact that the results obtained strongly depend on the choice of wet-lab workflow and the bioinformatics pipelines, as shown by our results. The scientific community must agree on the basic requirements to consider a dataset meaningful for both bioinformatic and biostatistics analysis. If such requirements would be clearly defined, then different workflows could be applied for the wet-lab part, as far as those requirements are achieved, exploring further protocols to reduce shotgun metagenomic sequencing costs and improving efficiency [38].

In this PT, the RNA viruses were hardly detected or not detected at all in the metagenomic datasets. There are different possible reasons for the observed failure to detect viruses. This is likely caused by the low relative spiking input of the viruses, in comparison to the final overall nucleic acid content, which was intended to resemble a situation of exogenous viral contamination. However, a pilot sequencing of a salmon aliquot after sample preparation with one-sixth $(10 \mu \mathrm{L})$ of the virus mix, resulted in a very low recovery for the viruses. Therefore, before the samples were sent out to the participants, another $50 \mu \mathrm{L}$ (five-sixth of the final amount of virus mix) was added to the frozen sample. Moreover, it should be mentioned that the necessary heat-inactivation of the virus mix could cause the virus particles to be destroyed, leading to a release of unprotected viral nucleic acids. Ultimately, this could have caused a loss of viral nucleic acids, before sample processing by the participants, due to exposure to the nucleases present in the salmon matrix. To solve the issue of low virus concentration, which is common to food and human samples, it was suggested that an RNA extraction be carried out and then to combine targeted with untargeted sequencing, spiking the variably sized panels $(100-10,000)$ of short primers into the reaction mixtures, at the reverse transcription step [39]. Following this approach, Thézé, J. et al., 2018 increased the number of Zika virus reads by more than tenfold, without substantially decreasing sensitivity for other pathogens in the metagenome. An enrichment during the library preparation, also allowed virus identification in foods like oysters [40].

Considering the relative abundances of all microorganisms of the mock community, the DNA metagenomic datasets were ranked according to their Bray-Curtis distance from the expected values. Although it was shown that MG-RAST has good sensitivity and precision when assigning reads to the species of a mock community [41], we acknowledge that MG-RAST suggests against the usage of the species taxonomic level, and that the bioinformatic processing of data might have affected the quantification of the species of the mock community, especially in the presence of possible contamination microorganisms that are close relatives of the mock community species. The DNA metagenomic ranking at the highest position (i.e., Bray-Cutis distance $<0.20$ ) were M36, followed by M38 characterized by 95 and $78 \%$ of the total reads assigned to the domain bacteria (Table 3), which were the main representatives of the mock community. In M36, the DNA was obtained using the QIAamp DNA Mini Kit (Qiagen) supplemented with proteinase K, the TruSeq Nano DNA Library Preparation protocol, and sequenced on an Illumina HiSeq2500 sequencer in rapid mode, at a read-length of $250 \mathrm{bp}$ paired-end to a coverage of $8.88 \mathrm{Gbp}$. With regards to the library preparation strategies, Grützke et al., 2019 [42] showed that genomes from bacterial species within a mock community were better detected using the TruSeq Nano, the Nextera Flex, and the TruPLEX kits over the Nextera XT kit. This result was explained with a shift in the GC density of fragments, generated with the Nextera XT kit to higher GC-contents, while this was balanced for the other three library kits, and reflected the expected distribution from the collection of mock-community reference genomes [41]. This specific issue was not investigated in this PT, although we also discovered a significant positive impact of the TruSeq Nano DNA Library Prep kit, on the quantification of S. aureus (Figure S3), which feature a genome with a low GC-content.

Among the variables investigated in the workflows, those impacting on the abundance of one or more microorganisms spiked in the salmon were the pre-processing protocol, the DNA extraction protocol, the library preparation strategy, and the sequencing platform. These results were certainly affected by the multi regressing modelling approach, quantifying the effect of each experimental 
variable (e.g., pre-processing) on the abundances of a certain species, when all other variables were fixed, which did not reflect what happened in real life, where each step of the wet-lab protocol impacts on the following. However, since each participant applied in house wet-lab protocols without restriction, the resulting variables in the metagenomic datasets were many, and the multi regressing modeling approach allowed us to collect meaningful results, species by species. This limitation might explain why sequencing coverage did not turn out to be a key variable affecting the relative abundance of the spiked organisms, although the six metagenomic datasets closest to the expected composition of the mock community were all sequenced at $\geq 8 \mathrm{Gbp}$. Clear indications on the sequencing coverage threshold to be achieved in metagenomic investigations are lacking. However, Ni et al., 2013 [43] stated that to detect bacteria species with a relative abundance of more than $1 \%$, a $20 \times$ coverage should be obtained, corresponding to a sequencing depth of $7.15 \mathrm{Gbp}$.

The investments in research on shotgun metagenomics are justified by the fact that the results achieved in both human and food sectors translate in the identification of potential pathogens in the ecosystems where those pathogens are in real life, and as a matter of fact, the interaction between pathogens and their ecosystems affects both pathogen survival and multiplication ability [2,44]. If sample preparation is designed to be as non-specific as possible to capture all nucleic acids regardless of their source, shotgun metagenomics is applicable simultaneously for viruses, bacteria, and parasites, as was found in this study for several workflows and according to previous reports [7]. In this framework, it is important to highlight that in the annual EFSA-ECDC report on foodborne and waterborne outbreaks occurring in the EU, there is always a high percentage of outbreaks for which the causative agent is described as 'unknown' or 'unspecified'. In the last available report, referring to the outbreaks which have occurred in 2018 in 28 Member States (MS) and 8 non-MS, such a percentage was as high as $23.8 \%$ [1] and some of these unknown were likely to be uncultivable or difficult to culture microorganisms, which could possibly be detected using shotgun sequencing. However, for diagnostic metagenomics to become truly useful, the method must provide robust and reproducible outputs [31].

In conclusion, our results showed that there are huge differences in the workflows applied in the wet-lab part of shotgun metagenomic sequencing at the international level, while there is a need for harmonized and validated protocols. Despite the differences between wet-lab protocols, all microorganisms of the mock community, belonging to the different domains (i.e., bacteria, parasite, yeast, DNA, and RNA viruses) were successfully detected in the metagenomic datasets obtained from spiked samples, suggesting that different wet-lab protocols could be successfully applied to reach the same result. Nonetheless, since the percentages of metagenomic datasets in which the mock community members were detected changed according to the number of reads selected as the detection cut-off level, this parameter should be clearly defined to use shotgun metagenomic sequencing in food microbiome studies, and in food safety risk assessment. There are many valuable papers on the application of shotgun metagenomics, but the lack of transparent information on the technical details of both the wet-lab and bioinformatic procedures are delaying the full implementation of this powerful sequencing approach.

Supplementary Materials: The following are available online at http://www.mdpi.com/2076-2607/8/12/1861/s1. Figure S1: Within-DNA workflow log-variance of the detected abundances. Figure S2: Box plots of the DESeq2 normalized read counts (detected abundances) obtained in group of samples defined by: (a) the categorized pre-processing procedure (i.e., categorized as protocols based on a beating protocol with TissueLyser (BBTL), no-processing (NO_PP), and other pre-processing protocols (OTHER_PP); (b) the DNA extraction protocol (i.e., DNeasy Power Food Microbial Kit (PowerFood), QIAamp DNA Mini Kit with or without SISPA and QIAamp UCP Pathogen Mini Kit, categorized as QIAamp, or the QIAamp Fast DNA Stool and DNeasy Power Soil, categorized as OTHER-EXD; (c) the library preparation strategy (i.e., Nextera ${ }^{\mathrm{TM}}$ XT DNA Library Prep kit and the Nextera ${ }^{\mathrm{TM}}$ DNA Flex Library Preparation kit, categorized as NexteraXT, and the NEBNext ${ }^{\circledR}$ Ultra ${ }^{\mathrm{TM}}$ II DNA Library Prep Kit for Illumina ${ }^{\circledR}$, TruSeq ${ }^{\circledR}$ DNA Library Prep Kit, GeneRead DNA Library kit other strategies, categorized as OTHER_L); (d) the sequencing platform (i.e., NextSeq500 and the HiSeq2500, categorized as NextSeq500, and the MiniSeq, MiSeq and Ion Torrent S5XL, categorized as OTHER_SP). Figure S3: Box plots of the mock community microorganisms' abundances (DESeq2 normalized counts) detected in the DNA workflows. Table S1: Relative abundance of the microorganisms of the mock community: Expected values and relative abundance quantified in the DNA metagenomic datasets. Table S2: Read counts of the microorganisms of the 
mock community in the tested metagenomes. The target nucleic acid is specified under the metagenomic dataset label. Table S3: Percentage of metagenomic datasets obtained from DNA and RNA in which each microorganism of the mock community was detected. Table S4: Ranking of the DNA metagenomic datasets in relation to their similarity to the expected composition of the mock community, considering bacteria only. Table S5: Comparison of the mock community microorganisms' abundances detected in the DNA metagenomic datasets when applying different pre-processing protocols, categorized as protocols based on a beating protocol with TissueLyser (BBTL), no-processing (NO_PP), and other pre-processing protocols (OTHER_PP). Table S6: Comparison of the mock community microorganisms' abundances detected in the DNA metagenomic datasets when DNA was extracted by the DNeasy Power Food Microbial Kit (PowerFood), QIAamp DNA Mini Kit with or without SISPA and QIAamp UCP Pathogen Mini Kit, categorized as QIAamp, or the QIAamp Fast DNA Stool and DNeasy Power Soil, categorized as OTHER-EXD. Table S7: Comparison of the mock community microorganisms' abundances detected in the DNA metagenomic datasets when using the Nextera ${ }^{\mathrm{TM}}$ XT DNA Library Prep kit and the Nextera ${ }^{\mathrm{TM}}$ DNA Flex Library Preparation kit, categorized as NexteraXT, or the NEBNext ${ }^{\circledR}$ Ultra $^{\mathrm{TM}}$ II DNA Library Prep Kit for Illumina ${ }^{\circledR}$, TruSeq $^{\circledR}$ DNA Library Prep Kit, GeneRead DNA Library kit other strategies, categorized as OTHER_L. Table S8: Comparison of the mock community microorganisms' abundances detected in the DNA metagenomic datasets, when using the NextSeq500 and the HiSeq2500, categorized as NextSeq500, and the MiniSeq, MiSeq and Ion Torrent S5XL, categorized as OTHER_SP. Table S9: Average abundance of each microorganism of the mock community detected in the spiked samples, by the workflows (WF), including DNA as target nucleic acid. Table S10: $t$-test adjusted $p$-values relative to the pairwise comparisons of the workflows (WF), including DNA as target nucleic acid.

Author Contributions: Designing and preparation of the mock community C.P., R.S.H., and S.J.P.; Sample preparation and shipping H.M.; Sample analysis and providing the shotgun metagenomics datasets J.G., A.B., T.N.P., P.D.C., F.C., R.J.E., C.A., M.H., S.L.G., G.M., J.M., A.N., C.R., and J.S. (Julien Schaeffer), J.S. (Joergen Schlundt), M.Y.F.T., R.S.H., S.J.P., and A.D.C.; Data analysis C.S., G.C., R.S.H., and S.J.P.; Study conceptualization C.S., R.S.H., S.J.P., and A.D.C.; Study coordination and writing of the paper C.S., and A.D.C.; Reviewing and editing of the paper C.S., J.G., P.D.C., F.C., R.J.E., G.C., M.H., S.L.G., G.M., J.M., C.R., J.S. (Julien Schaeffer), J.S. (Joergen Schlundt), F.M.A., R.J.E., S.J.P., and A.D.C. All authors have read and agreed to the published version of the manuscript.

Funding: This research was supported by the EU founded project COMPARE (Grant Agreement N ${ }^{\circ}$ 643476). J.G. was supported by a grant of the German Federal Ministry of Education, within the framework of the project Ess-B.A.R. (FKZ 13N13982).

Acknowledgments: Patricia König, Kerstin Wernike, and Bianka Hillmann are acknowledged for preparing the viruses for the mock community. We are grateful to Ulrich Nübel (DSMZ) and Sabine Gronow (DSMZ) for providing strain Propionibacterium freudenrichii DSM 20271.

Conflicts of Interest: The authors declare no conflict of interest.

\section{References}

1. European Food Safety Authority (EFSA). Scientific report on the European Union One Health 2018 Zoonoses Report. EFSA J. 2019, 17, 5926.

2. Yang, X.; Noyes, N.R.; Doster, E.; Martin, J.N.; Linke, L.M.; Magnuson, R.J.; Hua, Y.; Ifigenia, G.; Dale, R.W.; Kenneth, L.J.; et al. Use of metagenomic shotgun sequencing technology to detect foodborne pathogens within the microbiome of the beef production chain. Appl. Environ. Microbiol. 2016, 82, 2433-2443. [CrossRef]

3. Larsen, M.H.; Dalmasso, M.; Ingmer, H.; Langsrud, S.; Malakauskas, M.; Mader, A.; Trond Møretrø, T.; Sonja Smole Mozina, S.S.; Rychli, K.; Wagner, M.; et al. Persistence of foodborne pathogens and their control in primary and secondary food production chains. Food Cont. 2014, 44, 92-109. [CrossRef]

4. Aw, T.G.; Wengert, S.; Rose, J.B. Metagenomic analysis of viruses associated with field-grown and retail lettuce identifies human and animal viruses. Int. J. Food Microbiol. 2016, 223, 50-56. [CrossRef]

5. Leonard, S.R.; Mammel, M.K.; Lacher, D.W.; Elkins, C.A. Strain-level discrimination of Shiga toxin-producing Escherichia coli in spinach using metagenomic sequencing. PLOS ONE 2016, 11, e0167870. [CrossRef] [PubMed]

6. Kirstahler, P.; Bjerrum, S.S.; Friis-Møller, A.; La Cour, M.; Aarestrup, F.M.; Westh, H.; Pamp, S.J. Genomics-based identification of microorganisms in human ocular body fluid. Sci. Rep. 2018, 8, 4126. [CrossRef] [PubMed]

7. Wylezich, C.; Papa, A.; Beer, M.; Höper, D. A versatile sample processing workflow for metagenomic pathogen detection. Sci. Rep. 2018, 8, 13108. [CrossRef] [PubMed] 
8. Jagadeesan, B.; Gerner-Smidt, P.; Allard, M.W.; Leuillet, S.; Winkler, A.; Xiao, Y.; Chaffrong, S.; Van Der Vossenh, J.; Tangi, S.; Katasej, M.; et al. The use of next generation sequencing for improving food safety: Translation into practice. Food Microbiol. 2019, 79, 96-115. [CrossRef]

9. Lewandowska, D.W.; Zagordi, O.; Geissberger, F.D.; Kufner, V.; Schmutz, S.; Böni, J.; Metzner, K.J.; Trkola, A.; Huber, M. Optimization and validation of sample preparation for metagenomic sequencing of viruses in clinical samples. Microbiome 2017, 5, 94. [CrossRef]

10. Knudsen, B.E.; Bergmark, L.; Munk, P.; Lukjancenko, O.; Priemé, A.; Aarestrup, F.M.; Pamp, S.J. Impact of sample type and DNA isolation procedure on genomic inference of microbiome composition. MSystems 2016, 1. [CrossRef]

11. Jones, M.B.; Highlander, S.K.; Anderson, E.L.; Li, W.; Dayrit, M.; Klitgord, N.; Fabani, M.M.; Segutitan, V.; Green, J.; Pride, D.T.; et al. Library preparation methodology can influence genomic and functional predictions in human microbiome research. Proc. Natl. Acad. Sci. USA 2015, 112, 14024-14029. [CrossRef] [PubMed]

12. Tremblay, J.; Singh, K.; Fern, A.; Kirton, E.S.; He, S.; Woyke, T.; Lee, Y.; Chen, F.; Dangl, J.L.; Tringe, S.G. Primer and platform effects on $16 \mathrm{~S}$ rRNA tag sequencing. Front. Microbiol. 2015, 6, 771. [CrossRef] [PubMed]

13. Salter, S.J.; Cox, M.J.; Turek, E.M.; Calus, S.T.; Cookson, W.O.; Moffatt, M.F.; Turner, P.; Parkhill, J.; Loman, N.J.; Walker, A.W. Reagent and laboratory contamination can critically impact sequence-based microbiome analyses. BMC Biol. 2014, 12, 87. [CrossRef] [PubMed]

14. Hasan, M.R.; Rawat, A.; Tang, P.; Jithesh, P.V.; Thomas, E.; Tan, R.; Tilley, P. Depletion of human DNA in spiked clinical specimens for improvement of sensitivity of pathogen detection by next-generation sequencing. J. Clin. Microbiol. 2016, 54, 919-927. [CrossRef]

15. Forbes, J.D.; Knox, N.C.; Ronholm, J.; Pagotto, F.; Reimer, A. Metagenomics: The next culture-independent game changer. Front. Microbiol. 2017, 8, 1069. [CrossRef]

16. Lindgreen, S.; Adair, K.L.; Gardner, P.P. An evaluation of the accuracy and speed of metagenome analysis tools. Sci. Rep. 2016, 6, 19233. [CrossRef]

17. Brinkmann, A.; Andrusch, A.; Belka, A.; Wylezich, C.; Höper, D.; Pohlmann, A.; Nordahl Petersen, t.; Lucas, P.; Blanchard, Y.; Papa, A.; et al. Proficiency testing of virus diagnostics based on bioinformatics analysis of simulated in silico high-throughput sequencing data sets. J. Clin. Microbiol. 2019, 57, e00466-19. [CrossRef]

18. Narayanasamy, S.; Jarosz, Y.; Muller, E.E.; Heintz-Buschart, A.; Herold, M.; Kaysen, A.; Laczny, C.; Pinel, N.; May, P.; Wilmes, P. IMP: A pipeline for reproducible reference-independent integrated metagenomic and metatranscriptomic analyses. Gen. Biol. 2016, 17, 260. [CrossRef]

19. Treangen, T.J.; Koren, S.; Sommer, D.D.; Liu, B.; Astrovskaya, I.; Ondov, B.; Darling, A.; Darling, A.E.; Phillippy, A.M.; Pop, M. MetAMOS: A modular and open source metagenomic assembly and analysis pipeline. Gen. Biol. 2013, 14, R2. [CrossRef]

20. Petersen, T.N.; Lukjancenko, O.; Thomsen, M.C.F.; Sperotto, M.M.; Lund, O.; Aarestrup, F.M.; Sicheritz-Pontén, T. MGmapper: Reference-based mapping and taxonomy annotation of metagenomics sequence reads. PLoS ONE 2017, 12, e0176469.

21. Meyer, F.; Paarmann, D.; D'Souza, M.; Olson, R.; Glass, E.M.; Kubal, M.; Paczian, T.; Rodriguez, A.; Stevens, R.; Wilke, A.; et al. The metagenomics RAST server-A public resource for the automatic phylogenetic and functional analysis of metagenomes. BMC Bioinform. 2008, 9, 386. [CrossRef] [PubMed]

22. Kultima, J.R.; Coelho, L.P.; Forslund, K.; Huerta-Cepas, J.; Li, S.S.; Driessen, M.; Voigt, A.Y.; Zeller, G.; Sunagawa, S.; Bork, P. MOCAT2: A metagenomic assembly, annotation and profiling framework. Bioinformatics 2016, 32, 2520-2523. [CrossRef] [PubMed]

23. Minot, S.S.; Krumm, N.; Greenfield, N.B. One codex: A sensitive and accurate data platform for genomic microbial identification. BioRxiv 2015, 027607. [CrossRef]

24. Scheuch, M.; Höper, D.; Beer, M. RIEMS: A software pipeline for sensitive and comprehensive taxonomic classification of reads from metagenomics datasets. BMC Bioinform. 2015, 16, 69. [CrossRef] [PubMed]

25. Amid, C.; Pakseresht, N.; Silvester, N.; Jayathilaka, S.; Lund, O.; Dynovski, L.D.; Pataki, B.A.; Visontai, D.; Xavier, B.B.; Alako, B.T.; et al. The COMPARE Data Hubs. Database 2019, 2019, baz136. [CrossRef]

26. Allander, T.; Tammi, M.T.; Eriksson, M.; Bjerkner, A.; Tiveljung-Lindell, A.; Andersson, B. Cloning of a human parvovirus by molecular screening of respiratory tract samples. Proc. Natl. Acad. Sci. USA 2005, 102, 12891-12896. [CrossRef] 
27. Keegan, K.P.; Glass, E.M.; Meyer, F. MG-RAST, a metagenomics service for analysis of microbial community structure and function. In Microbial Environmental Genomics (MEG); Humana Press: New York, NY, USA, 2016; pp. 207-233.

28. Pruitt, K.D.; Tatusova, T.; Maglott, D.R. NCBI Reference Sequence (RefSeq): A curated non-redundant sequence database of genomes, transcripts and proteins. Nucl. Acids Res. 2005, 33 (Suppl. S1), D501-D504. [CrossRef] [PubMed]

29. McMurdie, P.J.; Holmes, S. phyloseq: An R package for reproducible interactive analysis and graphics of microbiome census data. PLoS ONE 2013, 8, e61217. [CrossRef]

30. Love, M.I.; Huber, W.; Anders, S. Moderated estimation of fold change and dispersion for RNA-seq data with DESeq2. Gen. Biol. 2014, 15, 550. [CrossRef]

31. Andersen, S.C.; Hoorfar, J. Surveillance of foodborne pathogens: Towards diagnostic metagenomics of fecal samples. Genes 2018, 9, 14. [CrossRef]

32. Benjamini, Y.; Hochberg, Y. Controlling the false discovery rate: A practical and powerful approach to multiple testing. J. R. Stat. Soc. Ser. B 1995, 57, 289-300. [CrossRef]

33. EFSA BIOHAZ Panel (EFSA Panel on Biological Hazards); Koutsoumanis, K.; Allende, A.; Alvarez-Ordonez, A.; Bolton, D.; Bover-Cid, S.; Chemaly, M.; Davies, R.; De Cesare, A.; Hilbert, F.; et al. Scientific Opinion on the whole genome sequencing and metagenomics for outbreak investigation, source attribution and risk assessment of food-borne microorganisms. EFSA J. 2019, 17, 5898.

34. Quince, C.; Walker, A.W.; Simpson, J.T.; Loman, N.J.; Segata, N. Shotgun metagenomics, from sampling to analysis. Nat. Biotechnol. 2017, 35, 833. [CrossRef] [PubMed]

35. Chiu, C.Y.; Miller, S.A. Clinical metagenomics. Nat. Rev. Genet. 2019, 20, 341-355. [CrossRef]

36. Ottesen, A.; Ramachandran, P.; Reed, E.; White, J.R.; Hasan, N.; Subramanian, P.; Ryan, G.; Jarvis, K.; Grim, G.; Daquiqan, N.; et al. Enrichment dynamics of Listeria monocytogenes and the associated microbiome from naturally contaminated ice cream linked to a listeriosis outbreak. BMC Microbiol. 2016, 16, 275. [CrossRef]

37. Walsh, A.M.; Crispie, F.; Daari, K.; O'Sullivan, O.; Martin, J.C.; Arthur, C.T.; Claesson, M.J.; Scott, K.P.; Cotter, P.D. Strain-level metagenomic analysis of the fermented dairy beverage nunu highlights potential food safety risks. Appl. Environ. Microbiol. 2017, 83, e01144-17. [CrossRef]

38. Baym, M.; Kryazhimskiy, S.; Lieberman, T.D.; Chung, H.; Desai, M.M.; Kishony, R. Inexpensive multiplexed library preparation for megabase-sized genomes. PLoS ONE 2015, 10, e128036. [CrossRef]

39. Thézé, J.; Li, T.; du Plessis, L.; Bouquet, J.; Kraemer, M.U.; Somasekar, S.; Yu, G.; de Cesare, M.; Balmaseda, A.; Kuan, G.; et al. Genomic epidemiology reconstructs the introduction and spread of Zika virus in Central America and Mexico. Cell Host Microbe 2018, 23, 855-864. [CrossRef]

40. Strubbia, S.; Schaeffer, J.; Oude Munnink, B.B.; Besnard, A.; Phan, M.V.T.; Nieuwenhuijse, D.F.; de Graaf, M.; Schapendonk, C.M.E.; Wacrenier, C.; Cotton, M.; et al. Metavirome Sequencing to Evaluate Norovirus Diversity in Sewage and Related Bioaccumulated Oysters. Front. Microbiol. 2019, 10, 2394. [CrossRef]

41. Peabody, M.A.; Van Rossum, T.; Lo, R.; Brinkman, F.S. Evaluation of shotgun metagenomics sequence classification methods using in silico and in vitro simulated communities. BMC Bioinform. 2015, 16, 362. [CrossRef]

42. Grützke, J.; Malorny, B.; Hammerl, J.A.; Busch, A.; Tausch, S.H.; Tomaso, H.; Deneke, C. Fishing in the Soup-Pathogen Detection in Food Safety Using Metabarcoding and Metagenomic Sequencing. Front. Microbiol. 2019, 10, 1805. [CrossRef] [PubMed]

43. Ni, J.; Yan, Q.; Yu, Y. How much metagenomic sequencing is enough to achieve a given goal? Sci. Rep. 2013, 3, 1968. [CrossRef] [PubMed]

44. Escobar-Zepeda, A.; Sanchez-Flores, A.; Baruch, M.Q. Metagenomic analysis of a Mexican ripened cheese reveals a unique complex microbiota. Food Microbiol. 2016, 57, 116-127. [CrossRef] [PubMed]

Publisher's Note: MDPI stays neutral with regard to jurisdictional claims in published maps and institutional affiliations. 